\title{
Prevalence and Associated Risk Factors of Uropathogenic Escherichia coli Isolates from Catheterized Persons at Ilorin Tertiary Hospital, Nigeria
}

\author{
Dele Ohinoyi Amadu ${ }^{1}$; Charles Nwabuisi ${ }^{1}$; Thairu Yunusa ${ }^{2}$; Idris Nasir \\ Abdullahi $^{3}$ : Janet Mosunmola Oladejo ${ }^{1}$; Ezekiel Seibu ${ }^{1}$; Abayomi Fadeyi ${ }^{1}$; \\ Yahaya Usman ${ }^{3}$; Dorcas Aliyu ${ }^{4}$ \\ ${ }^{I}$ Department of Medical Microbiology and Parasitology, University of Ilorin Teaching Hospital, \\ Ilorin, Kwara State, Nigeria \\ ${ }^{2}$ Department of Medical Microbiology and Parasitology, University of Abuja, Nigeria \\ ${ }^{3}$ Department of Medical Laboratory Science, College of Medical Sciences, Ahamdu Bello \\ University, Zaria, Nigeria \\ ${ }^{4}$ Department of Medical Laboratory Services, Family Health International 360, Abuja, Nigeria
}

Corresponding Author Idris Nasir Abdullahi

\section{Mobile: \\ $+2348022851352$ \\ E mail: \\ inabdullahi@abu.edu. $n g$}

Key words: Antimicrobial resistance, risk factors, ESBL, Biofilm
Background and study aim: Urinary tract infection (UTI) is among the most common complications of catheterized persons in hospital settings especially, those caused by extended spectrum beta lactamase (ESBL) and biofilm producing Escherichia coli.This hospital based, cross sectional study was carried out to determine the prevalence and associated factors associated with Uropathogenic Escherichia coli (UPEC) isolates from catheterized persons (inpatients and outpatients) attending Ilorin Tertiary hospital, Nigeria

Materials and Methods: Between $2^{\text {nd }}$ April and $30^{\text {th }}$ June 2016, urine samples from 113 catheterized inpatient and outpatients were evaluated. Female subjects accounted for $47(41.6 \%)$ of the study population. Standard microbiological methods and Analytical Profile Index (API) 20E system were used for the isolation and identification of UPEC, respectively. Tissue culture plate (TCP) technique was used to demonstrate biofilm production potentials.
Results: The prevalence of catheter associated urinary tract infection (CAUTI) in this study was $70.8 \%$ most of which are Gram negative bacilli belonging to the Enterobacteriaceae family with Escherichia coli $44(55.0 \%)$ being the most predominant pathogen. Extended spectrum beta lactamase (ESBL) $E$ coli in this study was $27.2 \%$ of which all $(100 \%)$ were strong biofilm producers. Female subjects had relatively higher prevalence of UPEC isolates, 29 $(65.9 \%)$ than the male counterparts. Whereas, the highest cases of UPEC was in 61-70 years age group, $29.5 \%$. There was significant association between UPEC and age and gender of subjects $(\mathrm{p}<0.05)$.

Conclusion: Findings from this study shows that $E$. coli is still the most common uropathogenic bacteria isolate in catheterized persons. Biofilm production confers some degree of ESBL production and antibiotic resistance.

\section{INTRODUCTION}

Some strains of E. coli are broadly categorized as extra intestinal pathogenic E. coli (EPEC) [1]. Uropathogenic $E$. coli (UPEC) has several virulence factors that allow it to colonize host mucosal surfaces, injure and invade host tissues, overcome hosts defense mechanisms and incite a host inflammatory response [1]. UPEC can colonize the bladder through the urinary tract and cause cystitis; this organism is also able to move through the ureters to the kidneys and cause pyelonephritis [2].

$E$ coli isolated from the urinary tract often express specific virulence properties that are not prevalent among isolates from normal fecal flora. The E.coli virulence factors (VFs) that cause urinary tract infection include adhesions, $\alpha$-hemolysin (Hly), cytotoxic necrotizing 
factor, fimbriae, aerobactin-mediated iron uptake, K1 capsular polysaccharide, and biofilm formation. These factors ultimately lead to tissue damage [3]. The ability of bacteria to attach to uroepithelial cells through specific fimbriae and adhesions is critical for the initiation of infection [4].

Biofilm formation is a dynamic process that can bring about wide variety of physiological events such as antibiotic tolerance, expression of virulence factors and increased resistance to host defense mechanisms [5]. Its emergence as an important ground of morbidity and mortality among hospitalized patients, visitors and staff is responsible for billions of dollars drained in treatment and work lost as such call for concern [5].

CA-UTIs are the most common nosocomial infections and a vast majority of them are caused by biofilm formed on urinary catheters. Catheterization and implanted medical devices will continue to increase especially with the aging population; hence the incidence of biofilm implicated infections will continue to rise [6]. Not many studies have documented biofilm formation especially amongst uropathogens in sub-Saharan Africa.

Catheterization and implanted medical devices will continue to increase especially with the aging population; hence the incidence of ESBL implicated $E$ coli infections will continue to rise [6]. Upsurge in antimicrobial resistance and the consequential complications of CA-UTI mediated by biofilms warrants a comprehensive understanding of the imperative role of specific urovirulence determinants especially the role of ESBL production in affected individuals. In view of these, the present study sought to determine the prevalence and associated risk factors of ESBL $E$ coli isolated from catheter urine of in- and outpatients attending University of Ilorin Teaching Hospital.

\section{MATERIALS AND METHODS}

\section{Study Area:}

This study was conducted at the Department of Medical Microbiology and Parasitology of the University of Ilorin Teaching hospital (UITH), Ilorin. UITH belongs to the second generation of Teaching Hospitals in Nigeria. It is a tertiary health care centre and the only referral centre in Kwara State with a capacity of over 450beds and an average of 10,000 to 12,000 annual admissions in-patient and out-patient's visits respectively in the last five years as captured by the Department of Health Information Management, UITH, in Ilorin, 2015. It is located in the North central region of Nigeria. The Hospital provides quality health care services to the neighboring states like Oyo, Kogi, Niger, Osun, and Ekiti states. Ilorin is the capital of Kwara state in Nigeria, West Africa. Ilorin coordinates on the globe at $8^{\circ} 30^{\prime} \mathrm{N}$ $4^{\circ} 33^{\prime} \mathrm{E}$. Ilorin's central location makes it easily accessible to all parts of the country. The infection control unit of UITH comprised of infectious diseases physicians, nurses, pharmacists and microbiologists. Infection prevention and control work on daily basis to prevent or control the spread of infections in the hospital wards, departments and units and the community. Clinicians suspecting occurrence of healthcare-associated infection (HCAI) report this to the Chief Medical Director or the Chairman, HCAICC (healthcare-associated infection control committee) or Infection control officer (ICO). All details regarding the patient, procedures, medication etc. are made available. Surveillance for HCAIs are prospectively monitored in high risk units of the hospital. High risk areas of the hospital include Operation Theatres (OT), Intensive care units (ICU), Transfusion services unit, Wards, Glutaraldehyde storage and monitoring unit, Kitchen/ Food handlers, Drinkable water source, Central Sterile Supply unit among others. Swabs of surfaces and air environment of OT are sampled and bacteriologically cultured once in six months. Sterilization are done in accordance to culture results. Samples (1ml) of in-use disinfectants, hand wash agents are taken and sent to the microbiology laboratory also once in 6 months for sterility analysis. Records of all microbiological analysis are kept with nurse in charge of OT and infection control nurse (ICN). In case of unacceptable results decision on corrective measures are taken by HCAICC.

\section{Sample Size Determination:}

Sample size was determined from a previous cross-sectional study by Fattahi [7], who reported prevalence of Biofilm forming UPEC as $92.0 \%$. With this, a sample size of 113 was calculated. Hence 113 study participants were enrolled.

\section{Study Design:}

This was a hospital-based cross sectional study. 


\section{Study Population:}

The study population comprises of catheterized in-patients and out-patients of all age groups and gender at clinics /units of the hospital such as General Outpatient Department (GOPD), Urology clinic, Male and Female Surgery wards, Accident and Emergency unit.

\section{Sampling Technique:}

Random sampling technique was employed for the selection of patients that met the inclusion criteria.

\section{Inclusion Criteria:}

1. Patients (both inpatient and outpatient) with indwelling catheter $\geq 48$ hours calendar days.

2. Those not on any antibiotics within the last 72 hours before recruitments.

\section{Exclusion Criteria:}

1. Patients (both inpatient and outpatient) with indwelling catheter $<24 \mathrm{hrs}$.

2. Those on antibiotics within 72 hours of enrollment in the study.

\section{Analytical Laboratory Procedures: \\ Catheter Urine Sample Collection and Transportation}

Urine sampling from patient with an indwelling urinary catheter was obtained from a sampling port using aseptic technique. Where there is no sampling port, the drain tubing was detached from the catheter bag and about $10-15 \mathrm{ml}$ urine is allowed to drain aseptically into a sterile receptacle. Collected urine in the catheter bag was not considered. All sample collection was carried with the assistance of an assigned Doctor. Samples collected were promptly transported to the Medical Microbiology and Parasitology department for analysis. However the samples that were unavoidably delayed were refrigerated at $2-8^{0} \mathrm{C}$ for not more than 4hours.

\section{Culture and Isolation:}

A modified semi-quantitative culture technique was used. Standard calibrated bacteriological loop (to determine the colony forming unit, $\mathrm{CFU}$ ) was used to aseptically transfer $0.001 \mathrm{ml}$ of a well mixed urine sample on appropriately well labeled culture of Cysteine Lactose Electrolyte Deficient (CLED) agar (Biomarker) and 5.0\% Sheep Blood Agar (Biomarker) media. All culture media were prepared according to the manufacturer's specifications. Incubation was at $37^{\circ} \mathrm{C}$ for $18-24$ hours aerobically.

\section{Characteristic of Isolates:}

E. coli appears on CLED as Large, elevated opaque yellow lactose fermenting colonies with a slightly deeper yellow center. E.coli appears on Blood Agar as large, opaque, sticky and colorless, \pm narrow clear hemolysis zone.

\section{Bacterial Biochemical Testing}

A Commercially available phenotypic qualitativ API 20E system (Bio Merieux, France) for Enterobacteriaceae identification was used for the biochemical confirmatory identification of UPEC isolates [8].

\section{Extended Spectrum beta Lactamase (ESBL) Detection:}

The double disk synergy test (DDST) using third generation cephalosporins (3GS) which includes ceftrazidime (30ug) and ceftriaxone (30ug) antibiotic discs alongside amoxicillin-clavulanate (10ug) disc were used for the detection of ESBL production amongst UPEC isolates. This was done by placing the antibiotic disk at distance of $20 \mathrm{~mm}$ from each other (center to center). Following incubation at $37^{\circ} \mathrm{C}$ aerobically for 18 24hrs, a positive synergistic effect showed inhibition zone between disks. A >5mm increase in a zone diameter for either antimicrobial agent tested in combination with clavulanic acid versus its zone when tested alone is taken as a positive ESBL production [8].

\section{Biofilm Assay Using Tissue Culture Plate (TCP) Method:}

A phenotypic quantitative Tissue Culture Plate (TCP) Method first developed and described by Christensen et al [10] and considered as the gold standard for biofilm detection was used for the detection of biofilm production in all UPEC isolates.

\section{Antibiotic Susceptibility Testing:}

This was performed according to the Clinical and Laboratory Standards Institute (CLSI), 2017 guidelines. In-vitro antibiotic susceptibility testing of established biofilm producing UPEC isolated was carried out by modified Kirby-Bauer disc diffusion method on Muller-Hinton (MH) agar plates. The following Oxoid ${ }^{\mathrm{TM}}$ antibiotic susceptibility disks (ThermoFisherTM, UK) were used; AmoxicillinClavulanate (10ug), Ceftriaxone (30ug), Ceftazidime $(30 \mu \mathrm{g})$, Ciprofloxacin $(5 \mu \mathrm{g})$, Gentamicin $(10 \mu \mathrm{g})$, Nitrofurantoin $(300 \mu \mathrm{g})$, and Imipenem (10ug). 
Quality Control:

International reference strains of Escherichia coli ATCC 25922 served as positive control.

\section{Questionnaire:}

Structured open ended questionnaires were prepared and administered on the spot to each participant. The participants were asked about sociodemographic data and clinical presentations/ diagnosis at enrollment for this study. Data accessed from questionnaires included their age, gender, marital status, education level, occupation, number of wives, sexual activity and places of resident.

\section{Statistical Analysis:}

The values obtained from this study were entered into a computer; data were analyzed using Statistical Package for Social Science (SPSS) version 21. Data were presented as bar-chart, pie chart and frequency tables. Chi-square test was used determine association between age, gender and UPEC isolates from subjects. $\mathrm{P}$ value $<0.05$ was considered as statistical significant.

\section{RESULTS}

Catheter urine samples from One hundred and thirteen (113) patients comprising of both inpatients and outpatients who met the inclusion criteria set out for this study were collected within the month of April -June, 2016.

Table 1: Sociodemographic characteristics of sampled population

\begin{tabular}{|c|c|c|}
\hline Variables & & Frequency Percentage \\
\hline \multirow[t]{3}{*}{ Residence } & a. Rural & 45.1 \\
\hline & b. Urban & 54.9 \\
\hline & Total & 100 \\
\hline \multirow[t]{3}{*}{ Sexual activity (last 10 days) } & a. No & 82.3 \\
\hline & b. Yes & 17.7 \\
\hline & Total & 113 \\
\hline \multirow{5}{*}{ Age } & $\leq 40$ & 11.5 \\
\hline & $\overline{4} 1-50$ & 4.4 \\
\hline & $51-60$ & 11.5 \\
\hline & $61-70$ & 40.7 \\
\hline & $>70$ & 31.8 \\
\hline \multirow[t]{2}{*}{ Gender } & Total & 100 \\
\hline & b. Male & $\frac{41.0}{58.4}$ \\
\hline \multirow{5}{*}{ Marital Status } & Total & 100 \\
\hline & a. Single & 3.5 \\
\hline & b. Married & 39.8 \\
\hline & c. Widow/ Widower & 56.6 \\
\hline & d. Total & 113 \\
\hline \multirow[t]{5}{*}{ Educational level } & a. No formal education. & 51.3 \\
\hline & b. Primary & 31.8 \\
\hline & c. Secondary & 21.2 \\
\hline & d. Tertiary & 1.7 \\
\hline & Total & 113 \\
\hline \multirow[t]{4}{*}{ Occupation status } & a. Unemployed & 40.7 \\
\hline & b. Employed & 23.0 \\
\hline & c. Self-employed & 36.3 \\
\hline & Total & 113 \\
\hline \multirow[t]{3}{*}{ Family structure } & a. Monogamous & 47.8 \\
\hline & b. Polygamous & 52.2 \\
\hline & Total & 113 \\
\hline
\end{tabular}

Male patients formed the majority of the sampled patients with 58.4\% while age group range of 61-70 $(40.7 \%)$ recorded the highest participants in this study. More of the subjects reside in urnan areas, 62 (54.9\%) resident than those from rural areas, 51 (45.1\%). Majority of the subjects had no sexual activity for the last 10 days before enrollment in the study, $93(82.3 \%)$. Widows/ widowers made the highest proportion of subjects, 64 (56.6\%). Majority of them were unemployed, 46 (40.7\%) (Table 1). 
Table 2: Clinical presentation of subjects enrolled for this study

\begin{tabular}{|l|c|c|}
\hline \multicolumn{1}{|c|}{ Clinical presentations } & Frequency & Percent (\%) \\
\hline Spinal Cord Injury & 1 & 9.0 \\
\hline Trauma Injury & 5 & 4.4 \\
\hline Unknown & 7 & 6.2 \\
\hline Neurogenic Bladder/retention (BPH) & 8 & 7.1 \\
\hline Bladder Outlet Obstruction (BOO) & 11 & 9.7 \\
\hline Incontinence & 20 & 17.7 \\
\hline Post-Operative Surgical Care & 23 & 20.4 \\
\hline Palliative Care for terminally ill/Old age & 38 & 33.6 \\
\hline \multicolumn{1}{|c|}{ Total } & 113 & 100.0 \\
\hline
\end{tabular}

As shown in Table 2, palliative care for terminally ill/old age patients had the highest frequency of $33.6 \%$ while patients with spinal cord injury had the least of $9.0 \%$.

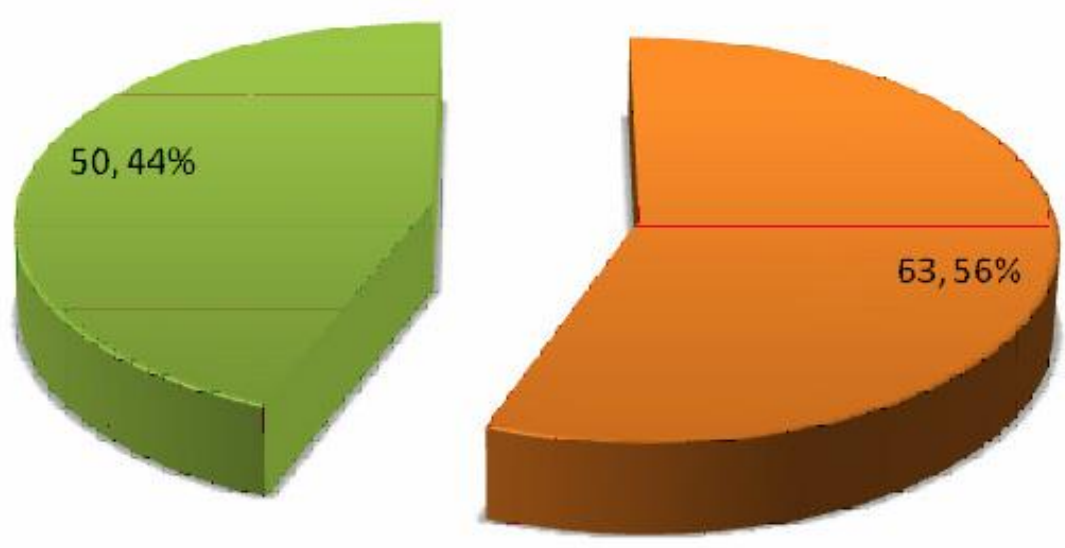

Figure 1: Pyuria level of the total sampled population

Microscopic examination of the catheter urine shows that $63(55.8 \%)$ recorded Pyuria level $\geq 10$ indicative of active infection as presented in Figure 1. 
Table 3: Cultural outcome of catheter urine after $24 \mathrm{hrs}$ of incubation at $37^{0} \mathrm{C}$

\begin{tabular}{|l|c|c|}
\hline \multicolumn{1}{|c|}{ Report } & Frequency & Percent \\
\hline Significant Pure culture isolates & 80 & 70.8 \\
\hline No growth (No visible colony) & 14 & 12.4 \\
\hline Mixed Growth (More than two isolates ) & 19 & 16.8 \\
\hline Total & 113 & 100.0 \\
\hline
\end{tabular}

As shown in Table 3, prevalence of CA-UTI was 80 (70.8\%) while those with mixed growth accounted for $19(16.8 \%)$.

Table 4: Relationship between gender and age amongst UPEC isolates

\begin{tabular}{|c|c|c|c|c|}
\hline \multirow[t]{2}{*}{ VARIABLES } & \multicolumn{2}{|c|}{ UPEC } & \multirow[b]{2}{*}{$\mathbf{X}^{2}$} & \multirow[b]{2}{*}{$\rho$} \\
\hline & $\begin{array}{c}\text { POSITIVE } \\
(\%)\end{array}$ & $\begin{array}{c}\text { NEGATIVE } \\
(\%)\end{array}$ & & \\
\hline \multicolumn{3}{|l|}{ GENDER } & \multirow{3}{*}{17.647} & \multirow{3}{*}{0.000028} \\
\hline Female & $29(65.9)$ & $18(26.1)$ & & \\
\hline Male & $15(34.1)$ & $51(73.9)$ & & \\
\hline \multicolumn{4}{|l|}{ AGE GROUPS } & \\
\hline$\leq 40$ & $11(25.0)$ & $2(2.9)$ & \multirow{5}{*}{17.647} & \multirow{5}{*}{0.0014} \\
\hline $41-50$ & $3(6.8)$ & $2(2.9)$ & & \\
\hline $51-60$ & $7(15.9)$ & $6(8.7)$ & & \\
\hline $61-70$ & $13(29.5)$ & $33(47.8)$ & & \\
\hline$\geq 71$ & $10(22.7)$ & $26(37.7)$ & & \\
\hline
\end{tabular}

Distribution of UPEC isolates across gender shows 29 (65.9\%) were females. While the highest occurrence across age groups were observed amongst $61-70$ years with $29.5 \%$. There was significant association between UPEC and age and gender of subjects $(\mathrm{p}<0.05)$.

Table 5: Distribution of UPEC isolates amongst patient's category

\begin{tabular}{|c|c|c|c|c|}
\hline \multirow[t]{2}{*}{ VARIABLE } & \multicolumn{2}{|c|}{ UPEC } & \multirow[b]{2}{*}{$\mathbf{X}^{2}$} & \multirow[b]{2}{*}{$P$} \\
\hline & $\begin{array}{c}\text { Positive } \\
(\%)\end{array}$ & $\begin{array}{c}\text { Negative } \\
(\%)\end{array}$ & & \\
\hline $\begin{array}{l}\text { Patients category } \\
\text { Inpatients }\end{array}$ & $32(72.7)$ & $22(31.9)$ & \multirow{2}{*}{17.963} & \multirow{2}{*}{0.000023} \\
\hline Outpatients & $12(27.3)$ & $47(68.1)$ & & \\
\hline
\end{tabular}

The occurrence of UPEC isolates was more frequent among Inpatients $32(72.7 \%)$ as shown in table 5. This was statistically significant association between frequency of UPEC isolated with patients' categories $(\mathrm{p}<0.05)$. 


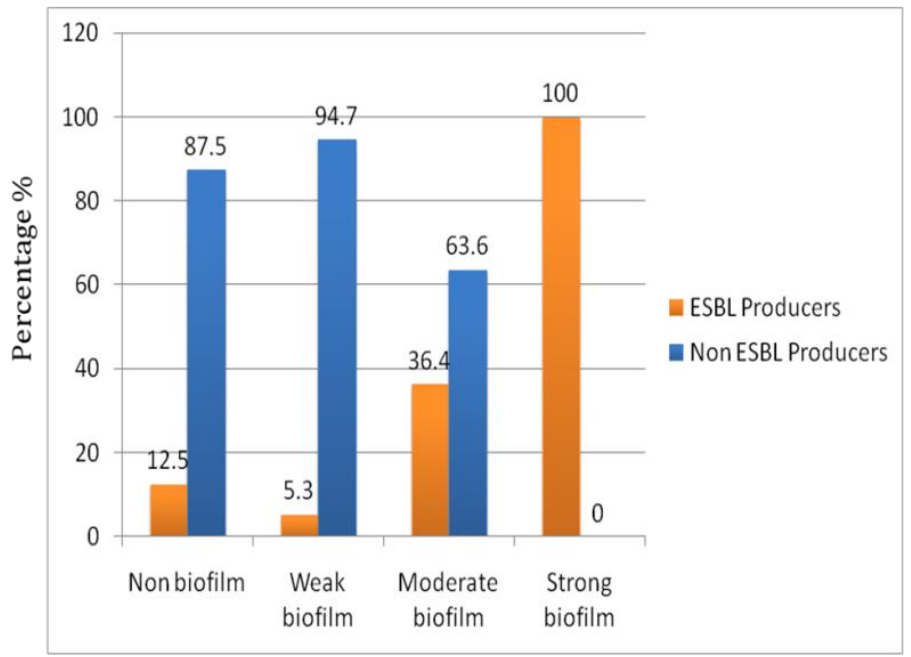

Degree of biofilm potentials

Figure 2: Relationship between biofilm and ESBL Production

Figure 2 shows a statistical significant difference $(p<0.05)$ in the incidence of ESBL across varied degree levels of biofilm formation. Highest incidence was observed in strong biofilm producers (100.0\%).

Table 6: Antibiotic Susceptibility Pattern of UPEC Isolates ( $\mathrm{n}=44)$

\begin{tabular}{|l|c|c|c|}
\hline \multicolumn{1}{|c|}{ Antibiotics } & Sensitive (\%) & Intermediate (\%) & Resistance (\%) \\
\hline Amoxicillin-Clavulanate & $13(29.54)$ & $0(0.0)$ & $31(70.45)$ \\
Ceftriaxone & $29(65.9)$ & $0(0.0)$ & $15(30.09)$ \\
Ceftazidime & $25(56.81)$ & $0(0.0)$ & $19(43.18)$ \\
Ciprofloxacin & $22(50.0)$ & $1(2.3)$ & $21(47.72)$ \\
Gentamicin & $6(13.64)$ & $0(0.0)$ & $38(86.36)$ \\
Nitrofurantoin & $31(70.45)$ & $0(0.0)$ & $13(29.54)$ \\
Imipenem & $41(93.2)$ & $3(6.8)$ & $0(0.0)$ \\
\hline
\end{tabular}

As shown in table 6, UPEC isolates exhibited high resistance to Gentamicin and AmoxicillinClavulanate of $86.36 \%$ and $70.45 \%$ respectively. Imipenem showed $100 \%$ effectiveness with no resistant UPEC isolate.

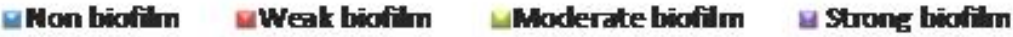

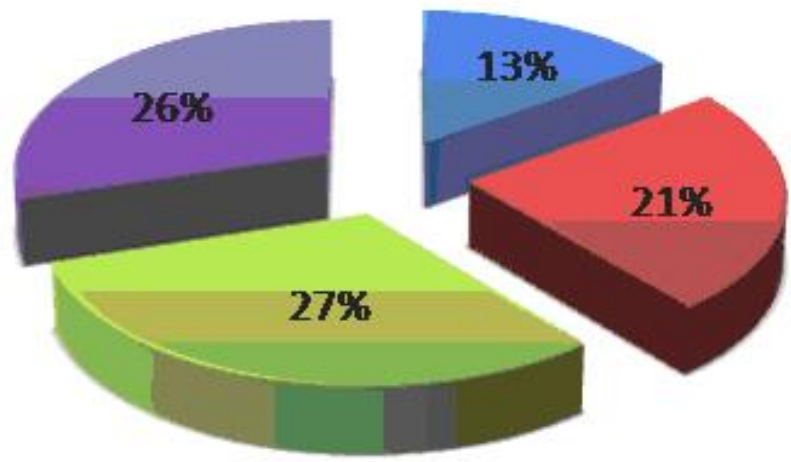

Figure 3: Resistance patterns and degree of biofilm formation

Average antibiotic resistance pattern amongst UPEC across the four grade levels of biofilm production showed Strong $26.0 \%$, Moderate $27.0 \%$, Weak $21.0 \%$ and Non biofilm producers $13.0 \%$ respectively. Non biofilm producing UPEC showed the least resistance pattern. 


\section{DISCUSSION}

This present study involved the recruitment and evaluation of 113 patients with indwelling urinary catheter, within the period of $2^{\text {nd }}$ April and $30^{\text {th }}$ June, 2016. These patients comprises of both outpatients and inpatients. The prevalence of CA-UTI in the study area is $(70.8 \%), 55.0 \%$ of which is caused by UPEC isolates. Prevalence of biofilm production potential by UPEC isolates was $31.8 \%$ respectively. Isolation and phenotypic identification of UPEC by standard microbiological methods and miniaturized API 20E system showed that $85.5 \%$ of the significant isolates observed in this study were gram negative bacilli belonging to the Enterobacteriacea. This observation is in agreement with Nicolle [10], who reported that most uropathogens are bacteria derived from the bowels that find their ways to the urinary tract through the urethra.

UPEC accounted for $44(55.0 \%)$ of the total uropathogens isolated, hence the most predominant pathogen. Comparatively the trio of Michael [11] and Nermeen et al [12] reported similar findings where UPEC was observed as the most prevalent uropathogen despite variation in the prevalence rates of $24.14 \%$ and $31.7 \%$ respectively. These variations may be partly explained by the differences in study populations and also in the inclusion criteria used by centers in selecting urine samples for culture. Antibiotic intake prior to presentation at the hospital may also be a key factor in bacterial yield. This study finding was however in contrast with Gruneberg [13] who posited that UPEC as the leading cause of urinary tract infections was being replaced by other members of the Enterobacteriaceae.

Distribution of UPEC across age groups confirmed that UPEC is common in all age groups. The highest frequency of $29.5 \%$ was observed amongst $61-70$ years while the least occurrence of $6.8 \%$ was observed amongst $41-50$ years. The high incidence as observed in advanced aged groups can be attributed to the presence of a number of risk factors some of which includes prostatic enlargements in males, diabetes mellitus, reduced ambulation, osteoporosis, interventional instrumentations like catheterization and weak bladder sphincter [14].

Further analysis shows that out of the total of 54(47.8\%) inpatients evaluated in this study, $32(72.7 \%)$ yielded significant growth of UPEC as summarized in Table 4.5, which was statistically significant $(\mathrm{p}<0.05)$. Similar observation was also reported by Ponnusamy and Nagappan [15] where $62.96 \%$ inpatients yielded UPEC isolates as compared to $37.03 \%$ from outpatients. Other previous studies which reported similar findings includes Hassan et al [16]; Jigna and Pratibha [17] who reported incidence rate of $83.3 \%$ and $60.0 \%$ respectively. There was significant statistical difference $(p<0.05)$ observed in the distribution of UPEC isolates across gender of which female subjects accounted for a notable frequency of $29(65.9 \%)$ despite higher male recruitment of 66(58.4\%). This finding was in line with other research such as that of Ponnusamy and Nagappan [17]; Kashef et al. [18] who reported prevalence in females as $56.0 \%$ and $60.0 \%$ respectively. This disparity between gender could be traceable to several factors which include anatomic differences such as the proximity of the female urethra to the anal region, hormonal effects and behavioral patterns.

Persistence of UPEC as a major ethological agent in UTI and as observed in this study and other similar research work can be attributed to the expression of a variety of virulence factors, which include adhesins (e.g., type 1 and P fimbriae) and toxins (e.g., haemolysin), cytotoxic necrotizing factor, fimbriae, aerobactin-mediated iron uptake, K1 capsular polysaccharide and biofilm formation [19].

Biofilm assay using the TCP method shows that the frequency of biofilm formation potentials amongst UPEC isolates in this study was $81.8 \%$. This finding was consistent with other studies such Niveditha et al [19] who reported $60.0 \%$ biofilm production rate respectively. Another study carried out by Ponnusamy et al [20] showed that among 100 E. coli strains, 72 (72.0\%) strains displayed a biofilm positive phenotype.

The occurrence rate of extended spectrum beta lactamase (ESBL) production of $27.2 \%$ amongst biofilm forming UPEC isolates in this study is a double barrel tragedy of public health concern which requires the prompt attention of caregivers. The highest occurrence of ESBL was $100.0 \%$ amongst the strong biofilm producers. This high incidence of ESBL reported in this study could be an indication of creation of selective drug pressure because of commonly use of cephalosporin and other antibiotics in our region [21].

UPEC isolates showed the highest susceptibility to antibiotics such as Imipenem $93.2 \%$ followed 
closely by nitrofuratoin with $70.45 \%$. The resistance pattern of UPEC isolates to the different antimicrobials agents employed in this study shows that among the antibiotic tested, resistance to gentamicin was the highest $(86.36 \%)$ while resistance to Imipenem was zero $(0.0 \%)$. Resistance to antimicrobial agents is well documented and has been noted since the first use of antibiotics which currently has become a worldwide problem [22]. This study finding reveals a higher antibiotic resistance development to the commonly used antibiotics for prophylaxis and for empirical therapy for UTI such as gentamicin and nitrofuratoin respectively. This may be due to increased consumption of these drugs, self-medication, and transfer of resistance isolates. These resistance pattern as observed is supported by the work of Hryniewicz et al [2] who stated that, the worldwide data is showing an increasing resistance among urinary tract pathogens urinary tract pathogens towards the conventional drugs.

\section{LIMITATION:}

This study is not without limitation, the major limitation of this study is enrolment of outpatients whom might have had the bacteria incubating in them before sampling. In addition, the inadequacy of sociodemographic variables studied. This might led more credence to risk factors data that favors contracting $E$ coli infection.

\section{CONCLUSION}

Findings from this study shows that $E$. coli is still the most common uropathogenic bacteria isolate in catheterized persons. Biofilm production confers some degree of ESBL production and antibiotic resistance.

\section{Conflict of interest:}

None

\section{Funding:}

Self funded

\section{Ethical Considerations:}

Ethical approval was sought from the University of Ilorin Teaching Hospital Ethical Review Committee (approval number: ERC/PAN/2016/1528). Written and/or verbal informed consent was also obtained from all participating subjects in accordance with the standards of human experimentation and with the Helsinki Declaration of 1975, as revised in 2000.
This was done via an informed consent forms duly completed by all the subjects.

\section{REFERENCES}

1. Soto SM, Smithson A, Martinez JA, Horcajada JP, Mensa J, Vila J. Biofilm formation in uropathogenic Escherichia coli strains: relationship with prostatitis, urovirulence factors and antimicrobial resistance. J Urol; 2007; 177(1):365-8.

2. Marrs CF, Zhang L, Foxman B. Escherichia coli mediated urinary tract infections:are there distinct uropathogenic E. coli (UPEC) pathotypes? FEMS Microbiol Lett. 2005; 252(2):183-90.

3. Dobrindt U. (Patho-)Genomics of Escherichia coli. Int J Med Microbiol. 2005; 295(6-7):

4. Terai A, Yamamoto S, Mitsumori K, Okada Y, Kurazono H, Takeda Y, Yoshida O. .Escherichia coli virulence factors and serotypes in acute bacterial prostatitis. Int J Urol. 1997; 4(3):289-94.

5. Gupta K. Increasing antimicrobial resistance and the management of uncomplicated community acquired urinary tract infections. J Antimicrob Agents. 2001; 135:41-50.

6. Kojic E.M. and Darouiche RO. Candida Infections of Medical Devices. Clinical Microbiology Review; 2004; 17 (2): 255-267.

7. Fattahi S, Kafil HS, Nahai MR, Asgharzadeh M, Nori R, Aghazadeh M. Relationship of biofilm formation and different virulence genes in uropathogenic Escherichia coli isolates from Northwest Iran. GMS Hyg Infect Control. 2015; 10 :Doc11.

8. Alonto A. Urinary Tract Infections. In: Textbook of Diagnostic Microbiology. C. L. Mahon, D. Lehman and G. Manuselis (eds.). 2007; pp. 1010-1029.

9. Christensen GD, Simpson, WA Younger JA. Adherence of coagulase negative staphylococci to plastic tissue cultures; A qualitative model for the adherence of staphylococci to medical devices. $J$ Clin Microbiol; 1995; 22:996 -1006.

10. Nicolle LE. Uncomplicated urinary tract infection in adults including uncomplicated pyelonephritis. Urol Clin North Am; 35:1-12.

11. Yakuku MN. Biofilm Production and Antibiotic Susceptibility of uropathogens isolated from Ahmadu Bello University Teaching Hospital Zaria, Nigeria. 2014; (Internet) available at abu.edu.ng (PDF) Accessed on the $1^{\text {st }}$ August, 2016.

12. Ahmed NM, Elsayed SB, Manal MY, El-gohary GM. Biofilm forming bacteria isolated from urinary tract infection, relation to catheterization and susceptibility to antibiotics. International $\mathrm{J}$. for Biotech and Mol. Bio. Res. 2011; 2(10):172178 . 
13. Gruneberg RN. Changes in urinary pathogens and their antibiotic sensitivities 1971- 1992. Journal of Antimicrobial Chemotherapy. 1994; 33, Suppl. A, 1-8.

14. Khan B, Saeed S, Akram A, Khan F, Nasim A. Nosocomial uropathogens and their antibiotic Sensitivity patterns in a tertiary referral teaching Hospital in rawalpindi, Pakistan. Journal of Ayub Medical College; 2010; 22 (1) 11- 12.

15. Ponnusamy P, Nagappan R. Extended spectrum beta-lactamase, biofilm-producing uropathogenic pathogens and their antibiotic susceptibility patterns from urinary tract infection-an overview. Int J Microbiol Res. 2013; 4 (2):1836-1842. 2544-2550.

16. Hassan A. Evaluation of Different Detection Methods of Biofilm Formation in the Clinical Isolates. Braz J Infect Dis. 2011; 15 (4), 2011, 305-311.

17. Jigna N, Pratibha D. Antibiotic resistance pattern in urinary isolates of Escherichia coli with special reference to extented spectrum Beta lactamases production. Inter J Phar Life Sci. 2012; 3: 14981502
18. Kashef N, Djavid GE, Shahbazi S. Antimicrobial susceptibility patterns of community- acquired uropathogens in Tehran, Iran. J Infect Dev Ctries. 2010;. 14 (4): 202-206

19. Niveditha S, Pramodhini S, Umadevi S, Kumar S, Stephen S. The Isolation and the Biofilm Formation of Uropathogens in the Patients with Catheter Associated Urinary Tract Infections (UTIs) J Clin Diag Res; 2010; 6(9): 1478-1482

20. Ponnusamy P, Nagappan R. Extended spectrum beta-lactamase, biofilm-producing uropathogenic pathogens and their antibiotic susceptibility patterns from urinary tract infection-an overview. Int J Microbiol Res. 2013; 4(2):1836-1842. 2544-2550.

21. Mittal S, Sharma M, Chaudhary U. Biofilm and multidrug resistance in uropathogenic Escherichia coli. Path Global Health; 2015; 109 (1):25-29

22. Hryniewicz K, Szczypa K, Sulikowska A, Jankowski K, Betlejewska K, Hryniewicz W. Antibiotic susceptibility of bacterial strains isolated from urinary tract infections in Poland. Journal of Antimicrob Chemother. 2001; 47: 773 - 80. 